\title{
Exploring the Feasibility of Electrostatic Shielding for Spacecrafts
}

\author{
R.K. Tripathi and J. W. Wilson \\ NASA Langley Research Center, Hampton, VA 23681 \\ R.C. Youngquist \\ Kennedy Space Center, FL 32899
}

\begin{abstract}
NASA is moving forward towards the agency's new vision for space exploration in the $21^{\text {st }}$ Century encompassing a broad range of human and robotic missions including missions to Moon, Mars and beyond. Exposure from the hazards of severe space radiation in deep space long duration missions is 'the show stopper.' Langley has developed state-of-the-art radiation protection and shielding technology for space missions. The payload penalty demands a very stringent requirement on the design of the spacecrafts for human deep space missions. The exploration beyond low Earth orbit (LEO) to enable routine access to more interesting regions of space will require protection from the hazards of the accumulated exposures of space radiation, Galactic Cosmic Rays (GCR) and Solar Particle Events (SPE), and minimizing the production of secondary radiation is a great advantage. There is a need to look to new horizons for newer technologies. The present investigation explores the feasibility of using electrostatic shielding in concert with innovative materials shielding and protection technologies. The asymmetries of the radiation shielding problem would be exploited in the electrostatics shielding process. The goal is to repel enough positive charge ions so that they miss the spacecraft without attracting thermal electrons. Conclusions are drawn about the advantages the electrostatic shielding, should it be successful, would bring to the radiation protection design process.
\end{abstract}

\section{Nomenclature}

$\begin{array}{ll}\mathrm{A} & =\text { number of nucleons } \\ D_{L} & =\text { dose } \\ \mathrm{Gy}-\mathrm{Eq} & =\text { gray equivalent } \\ \mathrm{E} & =\text { energy per nucleon in } \mathrm{MeV} \\ H & =\text { dose equivalent } \\ L E T & =\text { linear energy transfer } \\ Q(L) & =\text { quality factor } \\ \mathrm{RBE} & =\text { relative biological effectiveness } \\ \mathrm{Z} & =\text { charge number }\end{array}$

\section{Introduction}

On January 14, 2004, President George Bush set up a new vision for NASA. He articulated the agency's vision for space exploration in the 21st Century, encompassing a broad range of human and robotic missions including missions to Moon, Mars and beyond. As a result, there is a focus on long duration space missions. NASA is committed to the safety of the missions and the crew. There is an overwhelming emphasis on the reliability issues for space missions and the habitat. The cost effective design of the spacecraft demands a very stringent requirement on the optimization process. Exposure from the hazards of severe space radiation in deep space long duration missions is 'the show stopper.' Thus, protection from the hazards of severe space radiation is of paramount importance to the new vision. It is envisioned to have long duration human presence on Moon for deep space exploration. As NASA is looking forward to exploration in deep space, there is a need to go beyond current technology to the technology of the future. Faced with a limited budget and an expanding space exploration 
program, the old way of doing business is inadequate, and NASA requires revolutionary technologies to make advances.

An enabling technology for the exploration, the development, and the commercialization of space is a costeffective means of reducing the health risks from exposures to galactic cosmic rays (GCR) and a possible solar particle event (SPE). This has been a well-recognized challenge and a critical enabling technology for exploration in which astronaut health effects are of principal concern. The safety of missions and habitat is of prime concern with the development of space infrastructure and the eventual commercialization of space, as new materials and other space products are identified and as larger numbers of civilians become involved in space based careers. At the present stage of space exploration, the astronaut corps is a select group of individuals who normally enter into service near mid-life and have a very limited career duration that allows unusually high annual exposures during their short career. Even then, the mitigation of health risks is a great challenge. As we begin to build infrastructure for commercialization, the involvement of more ordinary career workers who will live and work in space will require a reassessment of allowable exposure limits and undoubtedly a substantial reduction in allowable annual exposure. Even more challenging is the "personal family explorer" who may choose to have a family vacation in space. The use of shielding to control exposure and the role of pharmacology in risk mitigation are critical issues in space development.

In the present paper, we will first review the underlying quantities to be considered and their implementation into the design process. We will then discuss the electrostatic radiation shielding and make conclusions about viable scenarios. Clearly, future developments will require a more complex mission scenario and optimization across a more complex array of habitats and vehicles.

\section{Shield Optimization}

Shield mass can be a high cost factor in system designs for the long-term operations required, and optimization methods in the design process will be critical to cost-effective progress in space development [1]. Limiting the time of transfer to a duty station or the mission time within the solar cycle as well as the choice of materials used in construction can reduce the shield mass required on specific missions [2]. Unfortunately, adequate optimization procedures have not been available to minimize the mass and the associated costs for a given mission scenario.

Much of the protection within a space structure is provided by the structural elements, onboard materials, and equipment required for other purposes and the means of making the best choice of materials among various options is critical to the protective qualities of the overall design. Multifunctionality of materials (for example, structural elements which have good shielding properties) will be common in the optimization process. Furthermore, the design decisions cannot be made in a vacuum and multidisciplinary design methods need to be developed. The need for multifunctional/multidisciplinary design techniques was identified as critical to the cost-effective development of space several years ago and expanded on recently.

In the past, an amount of exposure was assigned to each mission segment and developed as a subjective strategy with relative improvements of costs through material trades dependent on off-optimum design solutions. On the other hand, the necessary optimization methods for minimum mass determinations have been developed $[1,2]$ in performing trade studies to enable objective trade reduction costs by meeting exposure constraints over the entire mission architecture for each trade. In addition to optimized design trades, we have also considered the implementation of the principle of as low as reasonably a chievable (ALARA) required by federal regulation and normally ignored in mission design studies. The ALARA principle is met by added protection of the crew quarters where members spend a significant fraction of each day sleeping. The main crew quarter design is also used as the shelter from potential solar particle events during the mission. In this respect, an adequate strategy for exposure limitation during extra vehicular activity (EVA) activity is available, and the design is mainly the habitable volume and crew quarter/SPE shelter.

\section{A. Exposure and Other Constraints}

The present exposure constraints used in the space program are recommended for low Earth orbit (LEO) operations by the National Council on Radiation Protection (NCRP 2001) and approved by the NASA Administrator and the Occupational Safety and Health Administration (OSHA.) There are no limits for deep space operations due to the unusual composition of the GCR and the resultant uncertainties in associated health risks [2].

The NCRP did recommend that the limits for low earth orbit (LEO) operations could be used as a guide in deep space operational studies [3]. New exposure recommendations are now approved by the NCRP [3] and the new LEO limits are given the three critical organs of skin, ocular lens, and blood forming organ (BFO) in tables 1 and 2 
and are used recognizing the associated uncertainties. We use dose equivalent for the Gy-Eq since insufficient data will not allow Gy-Eq evaluation at this time.

The optimized mission is taken $[1,2]$ as the minimum mass to meet mission requirements and not exceed the exposure constraints in tables 1 and 2. The present design considerations are for the main habitable areas. The volume limited crew quarters where a large fraction of personal time is spent will have added protection to further reduce exposures (ALARA) and is also designed to provide the shelter from a solar particle event.

Table 1. Recommended organ dose equivalent limits for all

ages.

\begin{tabular}{|c|c|c|c|}
\hline & BFO, Sv & Eye, Sv & Skin, Sv \\
\hline Career & See Table 2 & 4.0 & 6.0 \\
\hline Annual & 0.50 & 2.0 & 3.0 \\
\hline 30 Days & 0.25 & 1.0 & 1.5 \\
\hline
\end{tabular}

Table 2. Career whole body-dose equivalent limit (Sv) for lifetime excess risk of fatal cancer of three percent as a function of age at exposure.

\begin{tabular}{|l|l|l|l|l|}
\hline Age & 25 & 35 & 45 & 55 \\
\hline Male & 0.7 & 1.0 & 1.5 & 2.9 \\
\hline Female & 0.4 & 0.6 & 0.9 & 1.6 \\
\hline
\end{tabular}

Aside from the radiation health risks, the psychological well being and its impact on crew performance also affects the shield design [1, 2]. Crew performance level is related in part to the length of the mission and the volume of the work/living areas of the spacecraft. The design performance levels of Optimal, Performance Limit, and Tolerable are used as a function of duration of the stay. Rather small volumes are useful over short time periods but long missions require sufficient space for a crew to perform at reasonable levels. We use the optimal design for the habitable volume and the Tolerable design for the crew quarters which also serves as the SPE shelter.

The basic requirement on astronaut exposure limitations established by the National Council on Radiation Protection is that radiation induced excess fatal cancer risks from career exposures are to be limited to be less than 3 percent and early radiation syndrome (nausea, vomiting...) is to be avoided $[3,10,11]$. Qualitatively, this is similar to the requirements for terrestrial radiation workers. The radiation environment in low Earth orbit (LEO) is of such a character that career radiation exposure limits have been given by the NCRP in terms of a local tissue related quantity known as dose equivalent (Seivert, NCRP 2001 [11]) given by

$$
H=\int Q(L) D_{L} d L
$$

where Q (L) is the quality factor (ICRP 1991) [12] relating to the difference in induced risk of differing particle types delivering the same dose and $\mathrm{D}_{\mathrm{L}}$ is the dose (Gray) from components with linear energy transfer between $\mathrm{L}$ and L + dL. Note that equation (1) breaks the convention of the ICRP (1991) [12] who have recommended radiation field weighting factors for estimation of fatal cancer risks, which does not depend on the local tissue field. The argument given by the ICRP is that the uncertainty introduced through such a nonlocal approximation is indicative of the uncertainty in risk estimation methods in distinction to equation (1) that gives the appearance of a quantified risk. The approach by the NCRP in recommending equation (1) allows a quantitative treatment of uncertainty as noted in reference [2] and enables the development of reliability based methods [13].

The RBE associated with early radiation syndrome have been recently defined by the NCRP (2001) [11] to relate to a new quantity Gy-Eq in terms of field quantities (nonlocal quantity) as

$$
\mathrm{Gy}-\mathrm{Eq}=\sum_{\mathrm{i}} \mathrm{RBE}_{\mathrm{i}} \mathrm{D}_{\mathrm{i}}
$$

where $R B E_{i}$ is the relative biological effectiveness of the ith field component resulting in dose $D_{i}$ to the specific tissue. Limitations on dose equivalent and Gray equivalent have been given by the NCRP for LEO operations as given in tables 1 and 2 . 


\section{B. Space Environment and Shielding Materials}

In order to quantify radiation exposure in space, it is required that the external ambient ionizing radiation environment be specified in terms of individual constituents and their respective energy fluxes. A great quantity of observational space environmental data from instrumented space platforms has been amassed in recent decades and used in developing computer models serving to define, as well as possible, the composition and temporal behavior of the space environment [4]. From the standpoint of radiation protection for humans in interplanetary space, the heavy ions (atomic nuclei with all electrons removed) of the galactic cosmic rays (GCR) and the sporadic production of energetic protons from large solar particle events (SPE) must be dealt with. The GCR environmental model used herein is based on a current version in which ion spectra are modulated between solar maxima and minima according to terrestrial neutron monitor data assuming the radial dependent diffusion model of Badhwar et al. [5], as described in reference [6]. The modeled spectra for Solar minimum in 1977 and Solar Maximum in 1990 as given by Badhwar are shown in figure 1 (left hand figure).

The environment near a large celestial body is modified by interaction with local materials producing an induced environment and shielding within the subtended angle of such a large body. The surface exposure on a lunar plain is shielded below the horizon but experiences an induced environment (mainly but not exclusively neutrons) produced in the local surface. The lunar surface GCR environment is shown in figure 1 (right hand figure) at the 1977 Solar Minimum and the 1990 Solar Maximum. In addition to the GCR ions streaming from overhead, large numbers of neutrons are produced in the lunar surface materials and diffuse from below the surface as shown in the figure. Similar results are obtained [1] on the surface of Mars. The main difference is the presence of the Martian atmosphere that attenuates the incident ions and produces additional GCR fragments and more energetic neutrons in the atmosphere overhead.
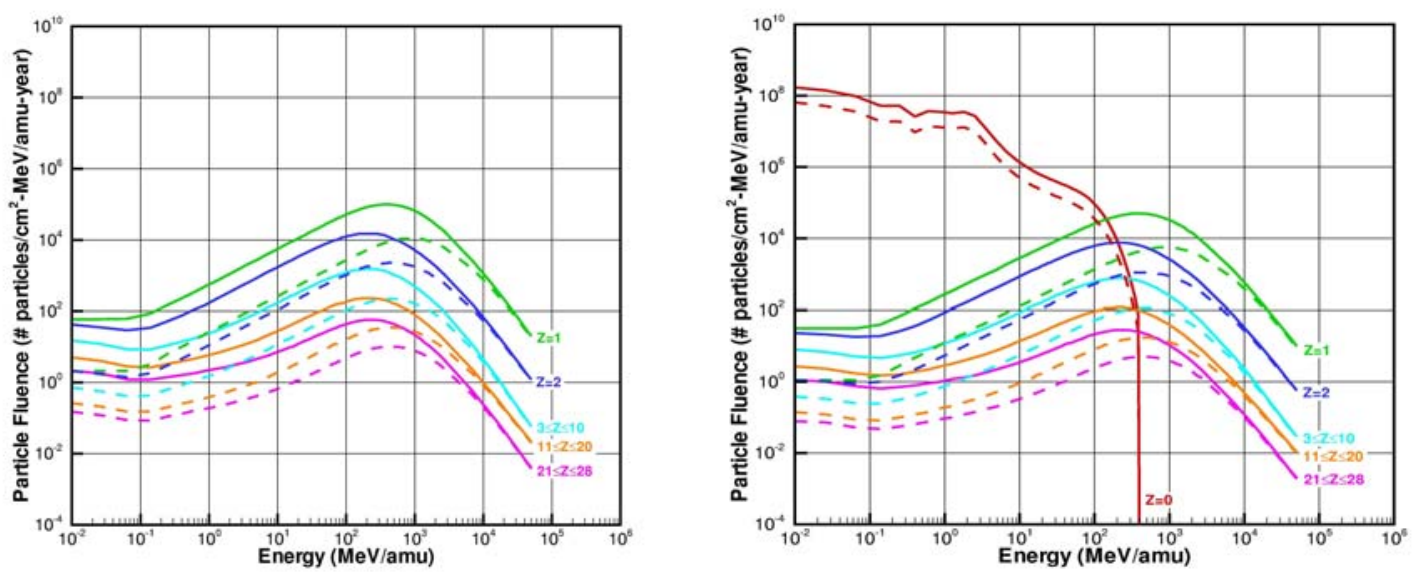

Fig. 1. Galactic cosmic ray spectra at the 1977 Solar Minimum (full lines) and 1990 Solar Maximum (dashed lines) according to Badhwar et al. Left figure free space, right figure on lunar surface.

In addition to the galactic cosmic rays able to penetrate the geomagnetic field to LEO, there are occasional solar particle events able to penetrate the geomagnetic field. The solar particle source is mainly composed of protons of similar quality as the trapped protons, and the limitations in tables 1 and 2 are applicable. The implications of the galactic cosmic ray exposures on LEO operations have not been fully evaluated with respect to exposure limitations.

Large SPE have only been observed to occur during times of increased solar activity conditions, and very large energetic events of grave importance to human protection occur only infrequently (avg. 1 or 2 per cycle) and only outside of two years of solar minimum. Among the large events, the largest observed ground level event of the last 60 years of observation is that of February 23, 1956 which produced a 3600 percent increase in neutron monitor levels on the terrestrial surface. The next largest event observed is the September 29, 1989 event with ground level increases of 400 percent or an order of magnitude smaller than that of Feb. 1956 event. Numerous other ground level events of smaller magnitude have occurred but are a factor of four and more lower in magnitude than the Sept. 1989 event. It is known that large SPEs are potentially mission threatening, and astronauts in deep space must have access to adequate shelter from such an occurrence. The SPE particle energy spectrum used here has been derived from the event which took place on September 29, 1989. To provide a baseline worst-case scenario, we assume an event of the order of four times larger than the September 29, 1989 event as an event comparable to the August 4, 1972 event from the point of view of space exposure. The September 1989 SPE spectrum is shown in figure 2. If 
we meet 30-day dose rate constraints on an event four times larger than the September 1989 event then it is unlikely that an added factor of two or so larger events (like that of Feb. 23, 1956) would have serious medical consequences.
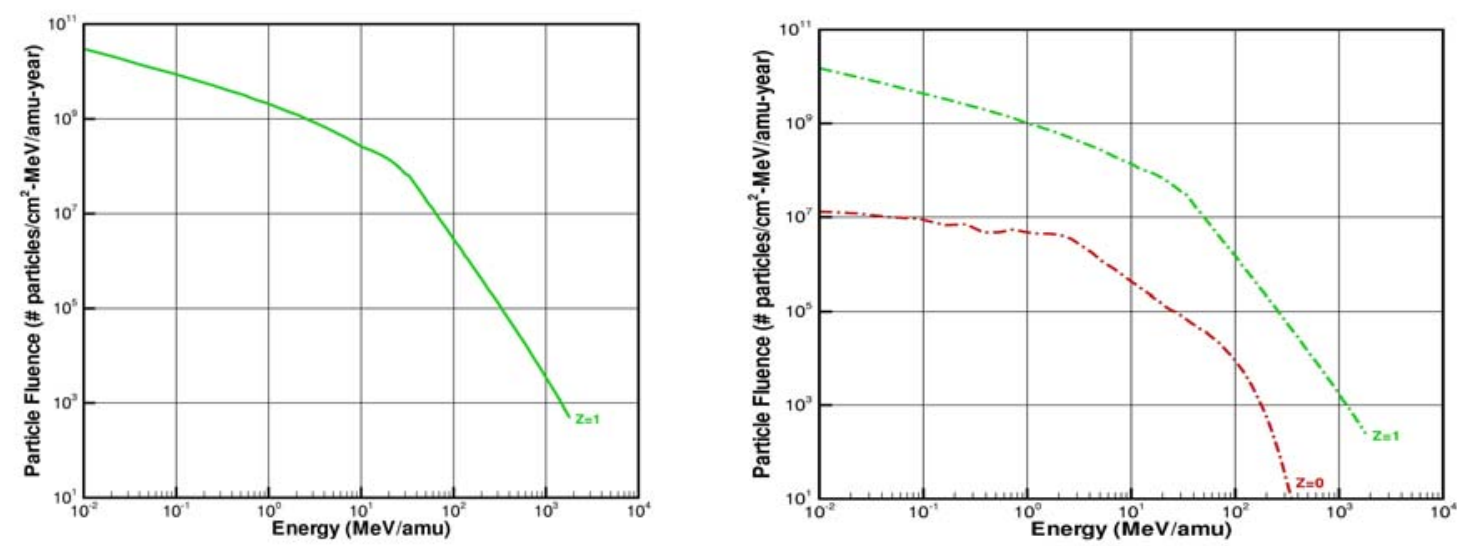

Fig. 2. Solar particle event September 1989, left figure free space and right figure Lunar surface.

The SPE are likewise altered by the presence of a large body similar to the GCR. The corresponding lunar surface environment is shown in figure 2. The role of the neutrons on the lunar surface is less effective in causing exposure relative to the protons streaming from overhead. Note that is in contrast to the more energetic GCR wherein large numbers of neutrons are produced in the lunar surface materials (see figure 1). Neutrons play a relatively more important role on the Martian surface where added neutrons are produced in the overhead atmosphere, and the SPE protons are greatly attenuated [1].

The effectiveness of a given shield material is characterized by the transport of energetic particles within the shield, which is in turn defined by the interactions of the local environmental particles (and in most cases, their secondaries) with the constituent atoms and nuclei of the shield material. These interactions vary greatly with different material types. Some of the materials used in the study at NASA Langley Research Center are given in table 3. For space radiation shields, materials with high hydrogen content generally have greater shielding effectiveness, but often do not possess qualities that lend themselves to the required structural integrity of the space vehicle or habitat. Organic polymers are the exception. The design of properly-shielded spacecraft and habitats for long-duration human presence in interplanetary space will thus require an approach tending toward optimization of a compromise between protective shielding and various other functional aspects of the onboard materials. Candidate multifunctional materials for such an optimization approach are chosen to represent various contributing elements in a vehicle shield design. Liquid hydrogen and methane are possible fuels that in large quantities may contribute substantially to overall protection. Aluminum has long been a spacecraft material of choice although various forms of polymeric materials show enhanced protection properties such as polyethylene. The polysulfone and polyetherimide are high performance structural polymers. Lithium hydride is a popular shield material for nuclear power reactors, but is generally not useful for other functions. The graphite nanofiber materials heavily impregnated with hydrogen may well represent a viable multifunctional component in future space structures, and its inclusion here should presently be considered as not yet state-of-the-art.

The results of detailed transport calculations for these materials have been incorporated into a shield design database. Important in this respect is their chemical composition and mass density given in table 3. The shield effectiveness for the above-discussed environments were evaluated using the Langley developed HZETRN code with improved neutron transport procedures [7]. The exposures to critical organ tissues were evaluated for each environment within spherical shells of each material. The time dependent dose rates are evaluated within the shielding materials assuming exposure in the center of a large volume with varying wall thickness. 
Table 3. Chemical composition of materials used in the present study

\begin{tabular}{|c|c|c|c|c|c|c|}
\hline Material & ID & Atom & $\mathbf{Z}$ & A & atoms/g & $\begin{array}{c}\text { Density } \\
\mathrm{g} / \mathrm{cm}^{2}\end{array}$ \\
\hline \multirow{6}{*}{$\begin{array}{c}\text { Aluminum } \\
2219\end{array}$} & ALM & $\mathrm{Al}$ & 13 & 27 & $2.08 E+22$ & 2.83 \\
\hline & & $\mathrm{Ti}$ & 22 & 48 & $7.53 E+18$ & \\
\hline & & V & 23 & 51 & $1.18 \mathrm{E}+19$ & \\
\hline & & $\mathrm{Mn}$ & 25 & 55 & $3.31 E+19$ & \\
\hline & & $\mathrm{Cu}$ & 29 & 64 & $5.90 E+20$ & \\
\hline & & $\mathrm{Zr}$ & 40 & 91 & $1.19 E+19$ & \\
\hline \multirow{4}{*}{$\begin{array}{c}\text { Poly- } \\
\text { etherimide }\end{array}$} & PEI & $\mathrm{H}$ & 1 & 1 & $2.44 \mathrm{E}+22$ & 1.27 \\
\hline & & C & 6 & 12 & $3.76 \mathrm{E}+22$ & \\
\hline & & $\mathrm{N}$ & 7 & 14 & $2.03 E+21$ & \\
\hline & & $\mathrm{O}$ & 8 & 16 & $6.10 E+21$ & \\
\hline \multirow[t]{4}{*}{ Polysulfone } & PSF & $\mathrm{H}$ & 1 & 1 & $3.00 E+22$ & 1.24 \\
\hline & & C & 6 & 12 & $3.68 E+22$ & \\
\hline & & $\mathrm{O}$ & 8 & 16 & $5.45 E+21$ & \\
\hline & & $\mathrm{S}$ & 16 & 32 & $1.36 \mathrm{E}+21$ & \\
\hline \multirow[t]{2}{*}{ Poly-ethylene } & PET & $\bar{H}$ & 1 & 1 & $8.60 E+22$ & 0.92 \\
\hline & & C & 6 & 12 & $4.30 E+22$ & \\
\hline \multirow{2}{*}{$\begin{array}{l}\text { Lithium } \\
\text { Hydride }\end{array}$} & $\mathrm{LIH}$ & $\mathrm{H}$ & 1 & 1 & $7.53 E+22$ & 0.82 \\
\hline & & $\mathrm{Li}$ & 3 & 7 & $7.53 E+22$ & \\
\hline \multirow{2}{*}{$\begin{array}{c}\text { Liquid } \\
\text { Methane }\end{array}$} & LME & $\mathrm{H}$ & 1 & 1 & $1.51 E+23$ & 0.466 \\
\hline & & C & 6 & 12 & $3.76 \mathrm{E}+22$ & \\
\hline \multirow{2}{*}{$\begin{array}{c}\text { Graphite } \\
\text { Nanofibers }\end{array}$} & GNF & $\mathrm{H}$ & 1 & 1 & $4.07 E+23$ & 2.25 \\
\hline & & C & 6 & 12 & $1.63 E+22$ & \\
\hline $\begin{array}{c}\text { Liquid } \\
\text { Hydrogen }\end{array}$ & $\mathrm{LH} 2$ & $\mathrm{H}$ & 1 & 1 & $6.03 E+23$ & 0.07 \\
\hline
\end{tabular}

\section{Electrostatic Shielding}

For the last four decades, investigations [8, 9] of the feasibility of using active methods, such as electromagnetic fields or plasmas, to shield spacecraft from hazardous space radiation, have been undertaken with the intention of reducing the weight penalties associated with the use of bulk material shielding for manned spacecraft. Most of 
these investigations have focused on high energy protons and electrons, that is more akin to SPE, and not much consideration was given to shielding against the high-energy, heavy-ions (HZE particles) present in the galactic cosmic ray (GCR) spectrum which are more biologically damaging. Amongst the four categories of active shielding; electrostatic fields, plasmas, confined magnetic fields and unconfined magnetic fields; it has been argued that unconfined magnetic fields concept is the most promising. The analysis suggested that electrostatic shields are unsuitable for GCR shielding since the required electrostatic potentials exceed the state of the art by over an order of magnitude. In addition, electrical breakdown considerations limit the minimum physical size of the shield configuration to dimensions on the order of hundreds of meters. Present feasibility investigation revisits the issue and considers both GCR and SPE space spectrum and takes into account all biological dose exposures.

\section{A. Shield Configuration and Transmission Function for Ions}

The electrostatic shield configuration considered here is shown in figure 3. It is composed of a set of 12 spheres (the center sphere represents a protected region within which is the spacecraft itself). The outer spheres are 20 meters in radius, located 160 meters along each axis, and at a potential of $-300 \mathrm{MV}$. The inner spheres are 10 meters in radius, located 50 meters along each axis and are at a potential of $+300 \mathrm{MV}$.

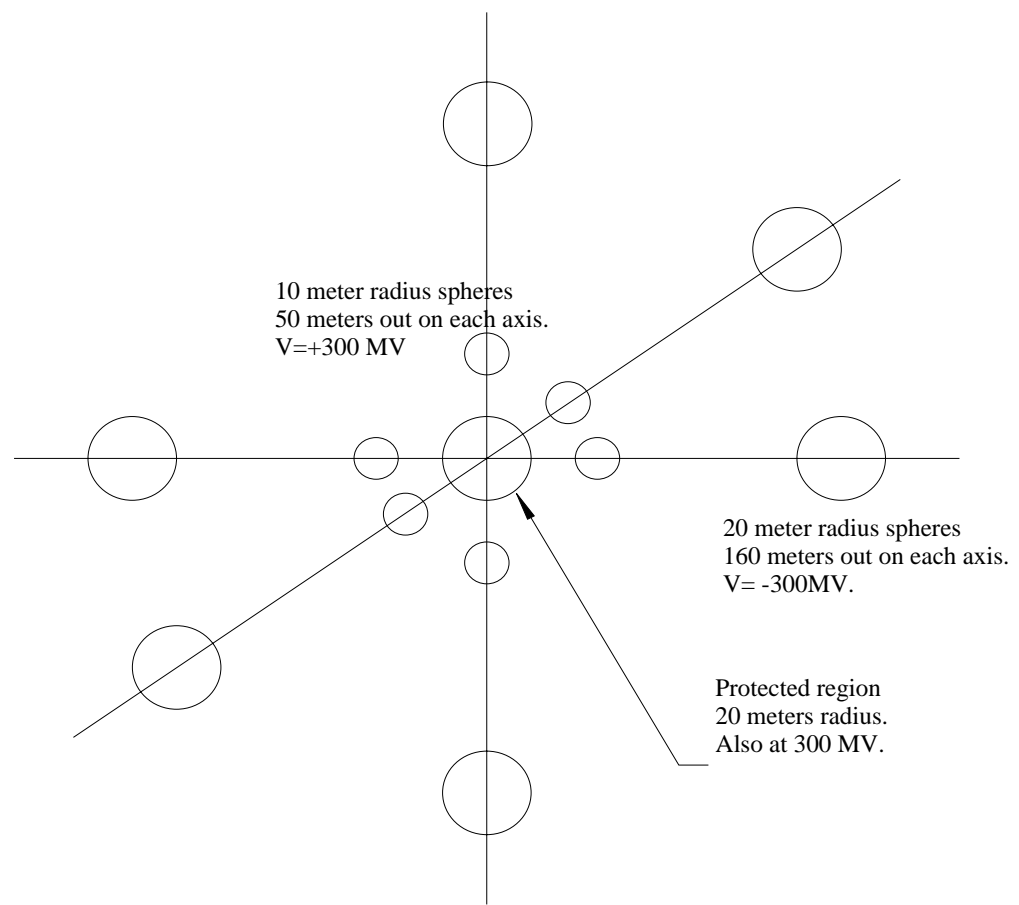

Figure 3: Electrostatic shielding configuration

Transmission of the shield for several ions (mainly proton and helium) was calculated for the configuration of figure 3. Further analysis suggested that equation (3) is a good representation of transmission for an ion.

$$
\text { Transmission }=1.36-\exp \{-((\mathrm{A} / \mathrm{Z}) \mathrm{E}-850) /((\mathrm{A} / \mathrm{Z}) \mathrm{E}+820)\}
$$

The notation "E" refers to the energy per nucleon in MeV, A is the number of nucleons in the ion and "Z" is the number of protons. Simulation studies for the transmission coefficient for the figure 3 configuration are shown in figure 4. The lower line is for protons and the upper one is for the case where $(\mathrm{A} / \mathrm{Z})=2$ where it is seen that the increased nucleon to charge ratio reduces the effectiveness of the shield.

Note also that particles that hit the protected area will be lowered in energy. For example, an ion with $6.0 \mathrm{GeV}$ initial kinetic energy that strikes the shield will have its energy reduced to $5.7 \mathrm{GeV}$ when it strikes. 


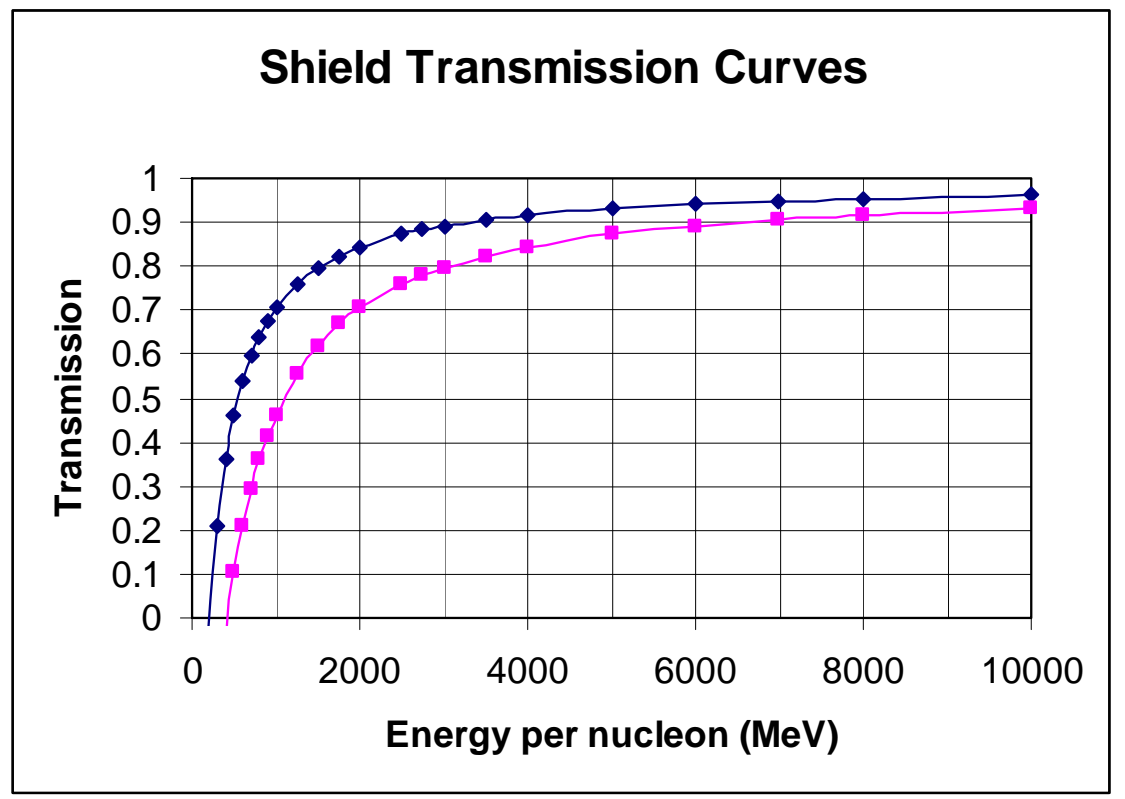

Figure 4: Transmission coefficient for the electrostatic shielding configuration

\section{B. Dose Exposure}

The results of detailed transport calculations for these transmission coefficients are shown in figure 5 . The dose exposures are for solar minimum environment of 1977 and consists of spectrum from proton (charge number, $Z=1$ ) to $\mathrm{Ni}(\mathrm{Z}=28)$ and takes into account 170 isotopes.

The blue (top) refers to the exposure for the full unmodified spectrum (electrostatic shielding turned off) and the pink (lower) curve refers to the spectrum with electrostatic shielding turned on that uses the transmission coefficients of equation (3). The percent reduction in dose exposure is about 70 percent at lower depths and about 84 percent at higher depths.

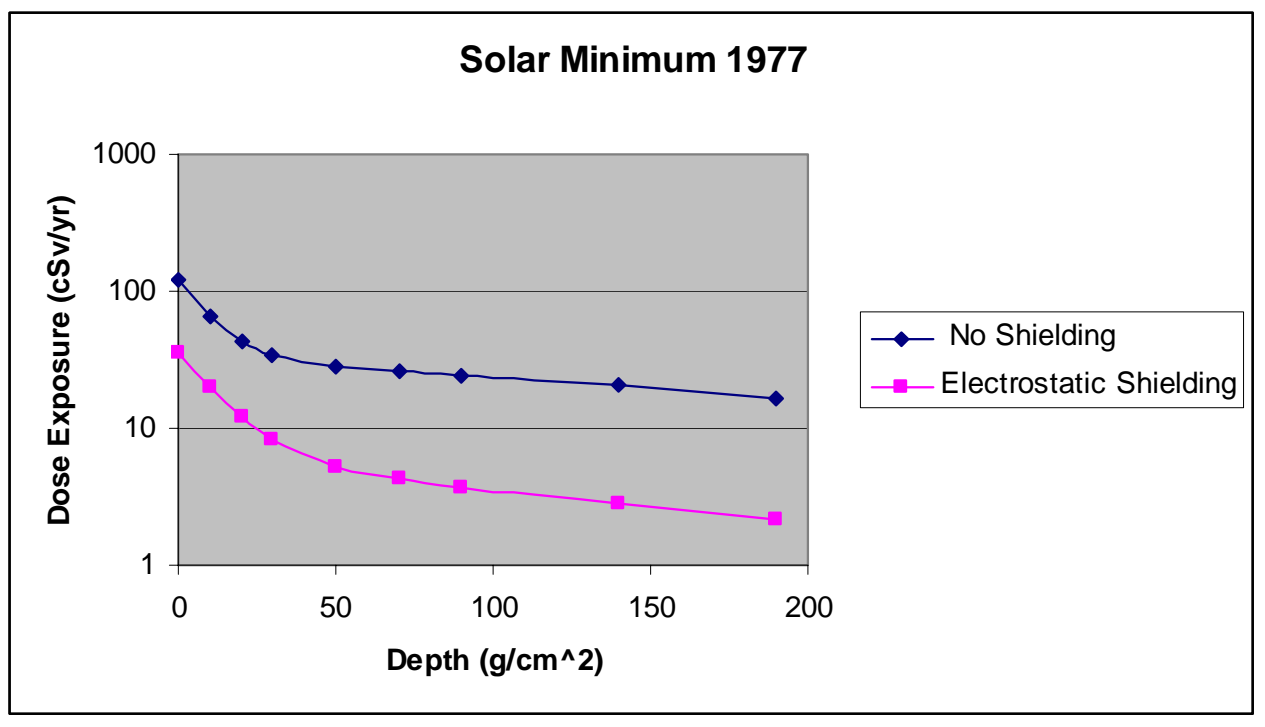

Figure 5: Dose exposure for electrostatic shielding 


\section{Comparison with material shielding}

Detailed analysis was used to make estimates of the radiation exposure using full solar minimum spectrum consisting of all ions from proton to nickel $(\mathrm{Z}=28)$ and includes 170 isotopes as discussed above. The blue (top) curve in figure 6 refers to the exposures from the unprotected (unmodified spectrum). The yellow (middle) curve refers to the exposure behind a polyethylene shielding and the pink (lowest) curve refers to the exposure with the modified spectrum with electrostatic shielding. As can be seen from the figure, the diversion of the ions from the spacecraft has an advantage. However, a spacecraft is made of materials; as a result, the selection of radiation protection shielding materials would always remain a vital part of the radiation protection strategy. Besides, multiple functional optimizations would always play an important role for the spacecraft architecture and shielding.

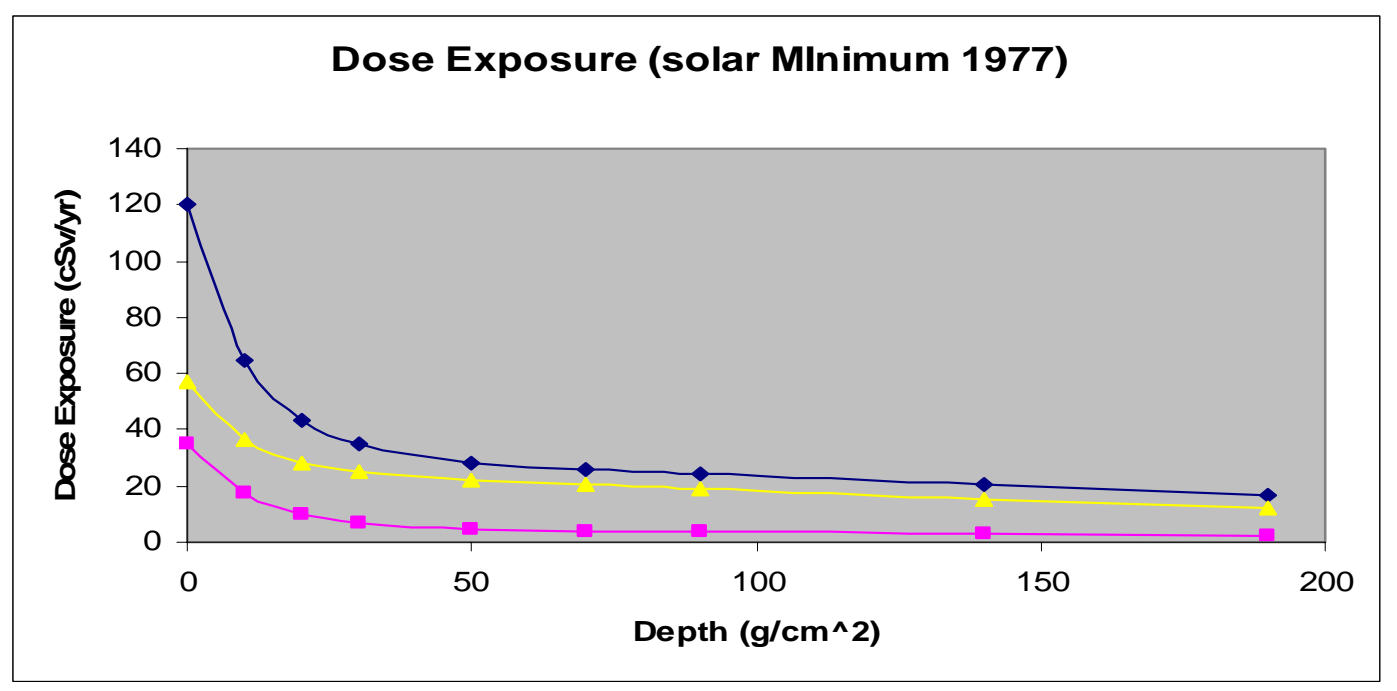

Figure 6: Dose exposure for electrostatic shielding and material shielding

\section{Conclusion}

Detailed analysis has been presented for a new configuration of electrostatic active shielding and comparison has been made with state-of-the-art material (shielding). It has been argued that material shielding and multifunctional optimization will always be an important ingredient of radiation protection and shielding. Electrostatic shielding has an advantage due to 'blocked' space radiation spectrum. However, technological feasibility of achieving the discussed configuration (or some other modified configuration) needs to be investigated. In the best case scenario, (should technologically active shielding be possible), a combination of the active and passive (material) shielding would be the best hope for radiation protection and shielding for the future direction.

\section{References}

${ }^{1}$ R.K. Tripathi, J.W. Wilson, F.A. Cucinotta, J.E. Nealy, M.S Clowdsley, M.Y. Kim, Deep space mission shielding optimization, SAE 2001-01-2326 (2001).

${ }^{2}$ R.K. Tripathi, L.C. Simonsen, J.E. Nealy, P.A. Troutman, J.W. Wilson, Shield optimization in simple geometry for the Gateway concept, SAE 2002-01-2332 (2002).

${ }^{3}$ NCRP, Guidance on Space Exposures in Space, National Council on Radiation Protection, Bethesda, NCRP Report No. 98 (1989).

${ }^{4}$ Badhwar, G.D. and O’Neill, P.M., Improved model of galactic cosmic radiation for space exploration mission, Nucl. Tracks \& Radiat., 20, 403-410, 1992.

${ }^{5}$ Badhwar G.D., et. al, Intercomparison of radiation measurements on STS-63; Radiat. Meas. 26; 147-158; 1997.

${ }^{6}$ Wilson, J.W., Kim M-H, Shinn, J.L., Tai, H., Cucinotta, F.A., Badhwar, G.D., Badavi, F.F., Atwell, W.: Solar Cycle Variation and Application to the Space Radiation Environment, NASA/TP-1999-209369.

${ }^{7}$ Clowdsley, M. S. et al., Can. J. Phys. 78: 45-56; 2000.

${ }^{8}$ Sussinham, J.C., Watkins, S. A., Cocks, F. H., Forty years of development of active systems for radiation protection of spacecrafts., J. Astronautical Sci 1999:47; 165-175.

${ }^{9}$ Townswnd, L.W., Overview of active methods for shielding spacecraft from energetic space radiation, International Workshop on Space Radiation Research and 't Annual NASA Space Radiation Health Investigators’ Workshop Arona (Italy), May 27-31, 2000. 
${ }^{10}$ NCRP, Uncertainties in Fatal Cancer Risk Estimates Used in Radiation Protection, NCRP Report No. 126 (1997).

${ }^{11}$ NCRP, Radiation Protection Guidance for Activities in Low-Earth Orbit, National Council on Radiation Protection, Bethesda, NCRP Report No. 132, (2001).

${ }^{12}$ ICRP, The 1990 Recommendations of the International Commission for Radiological Protection, ICRP Report 60, Pergamon Press, Oxford, UK, (1991).

${ }^{13}$ Tripathi, R.K. and Wilson, J.W., Enabling technology for safe human space missions, AIAA, Space 2004, paper 21652. 